\title{
Disruption of the Abdominal-B Promoter Tethering Element Results in a Loss of Long-Range Enhancer- Directed Hox Gene Expression in Drosophila
}

\author{
Margaret C. W. Ho ${ }^{1}$, Benjamin J. Schiller ${ }^{1}$, Omar S. Akbari ${ }^{1}$, Esther Bae ${ }^{2}$, Robert A. Drewell ${ }^{\text {* }}$
}

1 Biology Department, Harvey Mudd College, Claremont, California, United States of America, 2 College of Osteopathic Medicine of the Pacific, Western University of Health Sciences, Pomona, California, United States of America

\begin{abstract}
There are many examples within gene complexes of transcriptional enhancers interacting with only a subset of target promoters. A number of molecular mechanisms including promoter competition, insulators and chromatin looping are thought to play a role in regulating these interactions. At the Drosophila bithorax complex (BX-C), the IAB5 enhancer specifically drives gene expression only from the Abdominal-B $(A b d-B)$ promoter, even though the enhancer and promoter are $55 \mathrm{~kb}$ apart and are separated by at least three insulators. In previous studies, we discovered that a 255 bp cis-regulatory module, the promoter tethering element (PTE), located $5^{\prime}$ of the $A b d-B$ transcriptional start site is able to tether IAB5 to the $A b d-B$ promoter in transgenic embryo assays. In this study we examine the functional role of the PTE at the endogenous BX$C$ using transposon-mediated mutagenesis. Disruption of the PTE by $P$ element insertion results in a loss of enhancerdirected $A b d-B$ expression during embryonic development and a homeotic transformation of abdominal segments. A partial deletion of the PTE and neighboring upstream genomic sequences by imprecise excision of the $P$ element also results in a similar loss of $A b d-B$ expression in embryos. These results demonstrate that the PTE is an essential component of the regulatory network at the $\mathrm{BX}-\mathrm{C}$ and is required in vivo to mediate specific long-range enhancer-promoter interactions.
\end{abstract}

Citation: Ho MCW, Schiller BJ, Akbari OS, Bae E, Drewell RA (2011) Disruption of the Abdominal-B Promoter Tethering Element Results in a Loss of Long-Range Enhancer-Directed Hox Gene Expression in Drosophila. PLoS ONE 6(1): e16283. doi:10.1371/journal.pone.0016283

Editor: Johannes Jaeger, Centre for Genomic Regulation (CRG), Universitat Pompeu Fabra, Spain

Received August 18, 2010; Accepted December 21, 2010; Published January 21, 2011

Copyright: $\odot 2011$ Ho et al. This is an open-access article distributed under the terms of the Creative Commons Attribution License, which permits unrestricted use, distribution, and reproduction in any medium, provided the original author and source are credited.

Funding: The research in this paper was supported by funding to R.A.D. from the National Institutes of Health (NIH-HD54977) and the National Science Foundation (IOS-0845103) and a Howard Hughes Medical Institute Undergraduate Science Education Program grant (520051213) to the Biology department at Harvey Mudd College. M.C.W.H. received support from the Merck-American Association for the Advancement of Science (AAAS) Undergraduate Science Research Program. The funders had no role in study design, data collection and analysis, decision to publish, or preparation of the manuscript.

Competing Interests: The authors have declared that no competing interests exist.

*E-mail: drewell@hmc.edu

\section{Introduction}

To ensure a high fidelity of gene expression patterns in embryos a very strict functional requirement exists for the interaction of cisregulatory modules (CRMs) in the genome of animals during development $[1,2,3,4]$. Embryonic transcriptional enhancers, which direct specific spatio-temporal patterns of gene expression in the embryo, are not permitted to promiscuously activate transcription from non-target promoters. Chromatin structural organization, insulators and promoter competition are thought to play a role in regulating enhancer-promoter interactions $([5,6,7,8,9]$, for recent reviews see $[10,11,12])$. At the Drosophila bithorax complex (BX-C), an extensive network of CRMs located in over $300 \mathrm{~kb}$ of infraabdominal (iab) non-genic sequence is responsible for directing embryonic expression of just three homeotic genes; Ultrabithorax $(U b x)$, abdominal- $A(a b d-A)$ and $A b d o m i n a l-B(A b d-B)$ (Fig. 1) $[13,14]$. These three homeotic genes are critical for establishing cellular identities in the presumptive thoracic and abdominal segments during development [2,15].

We recently identified a $255 \mathrm{bp}$ promoter tethering element (PTE) located $5^{\prime}$ of the $A b d-B$ transcriptional start site that is responsible for specifically recruiting the IAB5 enhancer from the $\mathrm{BX}-\mathrm{C}$ to the $A b d-B$ promoter in competition assays on transgenes [16]. Furthermore, the PTE also demonstrates anti-insulator activity by enabling the IAB5 enhancer to bypass an insulator from the BX$\mathrm{C}$ to activate a target promoter on transgenes [17]. The ability of the PTE to facilitate specific enhancer-promoter interactions may explain how certain IAB enhancers, such as IAB5, IAB6, IAB7a and IAB $7 \mathrm{~b}[8,18,19]$, are able to bypass the Frontabdominal (Fab) insulators Fab-6, Fab-7 and Fab-8 [19,20,21,22] and direct transcription from the $A b d-B$ morphogenetic $(m)$ promoter at the endogenous BX-C (Fig. 1). As such, the PTE may be part of the complex regulatory network of CRMs, including the insulators, Promoter Targeting Sequences (PTSs) [23,24] and additional tethering sequences located $5^{\prime}$ of the $A b d-B$ gene $[25,26]$, responsible for mediating enhancer-promoter interactions in the BX-C. In contrast to the PTSs, which are located adjacent to the Fab-7 and Fab-8 insulators, the PTE is adjacent to the $A b d-B$ promoter (Fig. 1). The PTSs have been implicated in mediating insulator bypass for the IAB enhancers on transgenic constructs [23,24]. However, deletion of either of the characterized PTSs from the endogenous BX-C did not result in a significant phenotype $[8,18,19]$, suggesting that there might be other sequences capable of mediating the longrange enhancer-promoter specificity at the BX-C.

In this study, we demonstrate the functional importance of the PTE at the endogenous BX-C. Disruption of the PTE by a $P$ element insertion in vivo results in a loss of IAB enhancer-directed $A b d-B$ expression during development and a homeotic phenotype 


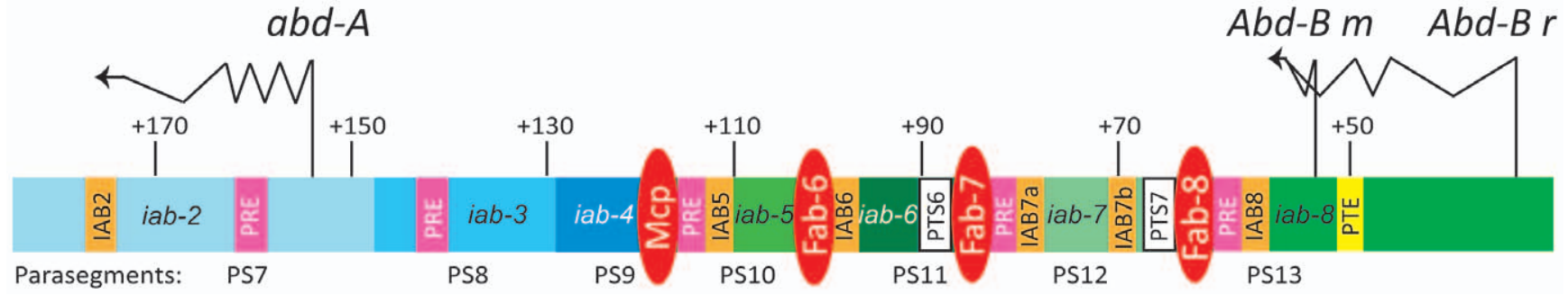

Figure 1. A network of interacting cis-regulatory modules control embryonic expression of abdominal- $A$ and $A b d o m i n a l-B$ in the Drosophila bithorax complex. The abd-A and Abd-B morphogenetic $(m)$ and regulatory $(r)$ transcripts are indicated by leftward arrows. The regulatory regions $i a b-2, i a b-3$ and $i a b-4$ (blue) interact with $a b d-A$. The $i a b-5, i a b-6$, iab-7 and $i a b-8$ regions (green) interact with $A b d-B$ m. Each $i a b$ region is thought to contain at least one characterized enhancer (orange rectangles) capable of directing Hox gene expression in a specific embryonic parasegments (PS). The positions of the characterized Fab-6, Fab-7, Fab-8 and Mcp insulators (red ellipses), promoter targeting sequence (PTS) modules (white rectangles), promoter tethering element (PTE) (yellow rectangle) and polycomb response elements (PREs) (pink rectangles) are indicated. Numbers above line refer to kilobase positions in DNA sequence accession number: DM31961. doi:10.1371/journal.pone.0016283.g001

when placed in hemizygosity with an $A b d-B$ null allele. Further molecular analysis of a mutant generated by imprecise excision of the $P$ element reveals that a partial deletion of the PTE and neighboring upstream genomic sequences results in a similar loss of enhancer-directed $A b d-B$ expression. These results demonstrate the critical functional role that the PTE plays in regulating promoterenhancer interactions at the endogenous Drosophila BX-C.

\section{Results}

Insertions at the PTE disrupt $A b d-B$ expression and result in homeotic transformation

Genetic studies were carried out to determine whether the activity of the PTE is important for in vivo IAB enhancer $-A b d-B$ interactions in the context of the BX-C. The previously identified $A b d-B^{T 2 \mathcal{N}}$ mutation [27] is derived from a $P$ element insertion in the endogenous PTE sequence $(-241$ bp relative the $A b d-B$ transcription start site). The original $P$ element insertion line $\left(A b d-B^{L a c 1}\right)$ contains a $5.6 \mathrm{~kb} A b d-B$ promoter region which includes $4.3 \mathrm{~kb}$ of $5^{\prime}$ sequence harboring the PTE sequence, fused to a lac $Z$ reporter gene. This $P$ element also contains the Fab- 8 insulator element, an IAB 8 enhancer element and the rosy gene. In contrast, the derived $A b d-B^{T 2 \mathcal{N}}$ line contains only a truncated $A b d-B$ promoter-lac $Z$ reporter gene, generated by imprecise excision of the $5^{\prime}$ region of the $P$ element (Fig. 2a). In the $A b d-B^{T 2 N}$ line the $P$ element is inserted within the endogenous $255 \mathrm{bp}$ PTE sequence, while leaving the entire $A b d-B$ morphogenetic $(m)$ transcription unit intact [27]. The $A b d-B^{T 2 \mathcal{N}}$ mutant is described as a Class I $A b d-B$ mutant, capable of complementing mutations in the $A b d-B$ regulatory $(r)$ transcript, but affecting $A b d-B m$ transcript expression [27]. In hemizygosis with the $A b d-B^{M 1}$ null allele, the $A b d-B^{T 2 \mathcal{N}}$ insertion line shows a phenotypic transformation of abdominal segments 5-8 towards a more anterior abdominal segment identity. This is seen most clearly in the complete transformation of the seventh abdominal tergite to a more anterior identity in the $A b d-B^{T 2 N}$ insertion line (Fig. 2c) when compared to wild-type (WT) (Fig. 2b). The homeotic transformation is easiest to see in the cuticles of adult females due to their lighter abdominal pigmentation when compared to males (Fig. 2b and c), although it is apparent in both sexes. This phenotype is characteristic of $A b d-B m$ mutations [27,28], suggesting that the $P$ element insertion into the PTE is disrupting expression from the adjacent $A b d-B m$ promoter and is consistent with a reduction in IAB5-7 enhancer- $A b d-B$ interactions.

In WT D. melanogaster embryos, the expression pattern of the $A b d-B m$ transcript extends from parasegment (PS) 10-13 during the germ-band elongation stage of development, while the $r$ transcript is predominantly restricted to PS14 at this stage [29,30].
In situ hybridization with an RNA probe that detects both the $m$ and $r$ transcripts in germ-band elongation stage embryos generated from crosses of heterozygous balanced $A b d-B^{T 2 \mathcal{N}}$ adults (Fig. 2f) [27], demonstrates a loss of $A b d-B$ expression in PS10-12 (presumptive abdominal segments 5-7) when compared with expression in WT embryos (Fig. 2d). These results indicate that the transposon insertion in the PTE impairs the ability of the IAB5-7 enhancers in the BX-C to drive expression from the endogenous $A b d-B m$ promoter. The detectable expression of $A b d-B$ in PS13 in $A b d-B^{T 2 N}$ embryos raises the question of why there is a homeotic transformation of the eighth abdominal segment in hemizygous $A b d-B^{T 2 \mathcal{N}} / A b d-B^{M 1}$ adults. While this phenotype is significant, it is only a partial transformation of A8 towards a more anterior identity (Fig. 2c), suggesting that there is not a complete loss of $A b d-B$ patterning function. It is possible that there may be subtle modulation of the level of $A b d-B$ gene expression in PS13 which, while responsible for the phenotypic transformation, is not readily detectable using RNA in situ hybridization. The expression of $A b d-B$ persists in PS13 and 14 at least through stage 13 of development (Fig. $2 \mathrm{~h}$ and i). However, it is also possible that transcription of $A b d-B$ in late embryonic or larval stages may be modulated from the $A b d-B^{T 2 \mathcal{N}}$ allele.

Knowing that expression from the endogenous $A b d-B m$ promoter was perturbed in $A b d-B^{T 2 \mathcal{N}}$ mutants, we examined whether the enhancers from the BX-C were re-directed to the intact ectopic $A b d-B m$ promoter (containing the PTE sequence), which drives lac $Z$, on the $P$ element insertion. In $A b d-B^{T 2 \mathcal{N}}$ embryos, lac $Z$ expression was detected in a pattern extending from PS10 to 14 (Fig. 2g). The lac Z expression appears strongest in PS13 (presumptive abdominal segment 8) and weaker in PS10 (Fig. 2g), presumably due to the proximity of the endogenous IAB8 regulatory sequences to the target promoter, as previously observed [27]. This pattern of lacZ expression is consistent with the $A b d-B$ expression pattern detected in wild-type embryos (Fig. 2d) and indicates that the IAB enhancers from the endogenous BX-C are now being re-directed to the intact ectopic $A b d-B$ promoter (containing the PTE) on the $P$ element to drive expression of lac $Z$ in $A b d-B^{T 2 N}$ embryos.

\section{Deletion of PTE and neighboring upstream sequences} results in a loss of enhancer-directed $A b d-B$ transcription

In the $A b d-B^{T 2 \mathcal{N}}$ mutant endogenous PTE function is disrupted by $P$ element insertion. In order to address the loss-of-functional activity of the PTE more directly we generated a mutant line in which the $5^{\prime}$ portion of the PTE and neighboring upstream genomic sequences are deleted from the endogenous BX-C locus 
A
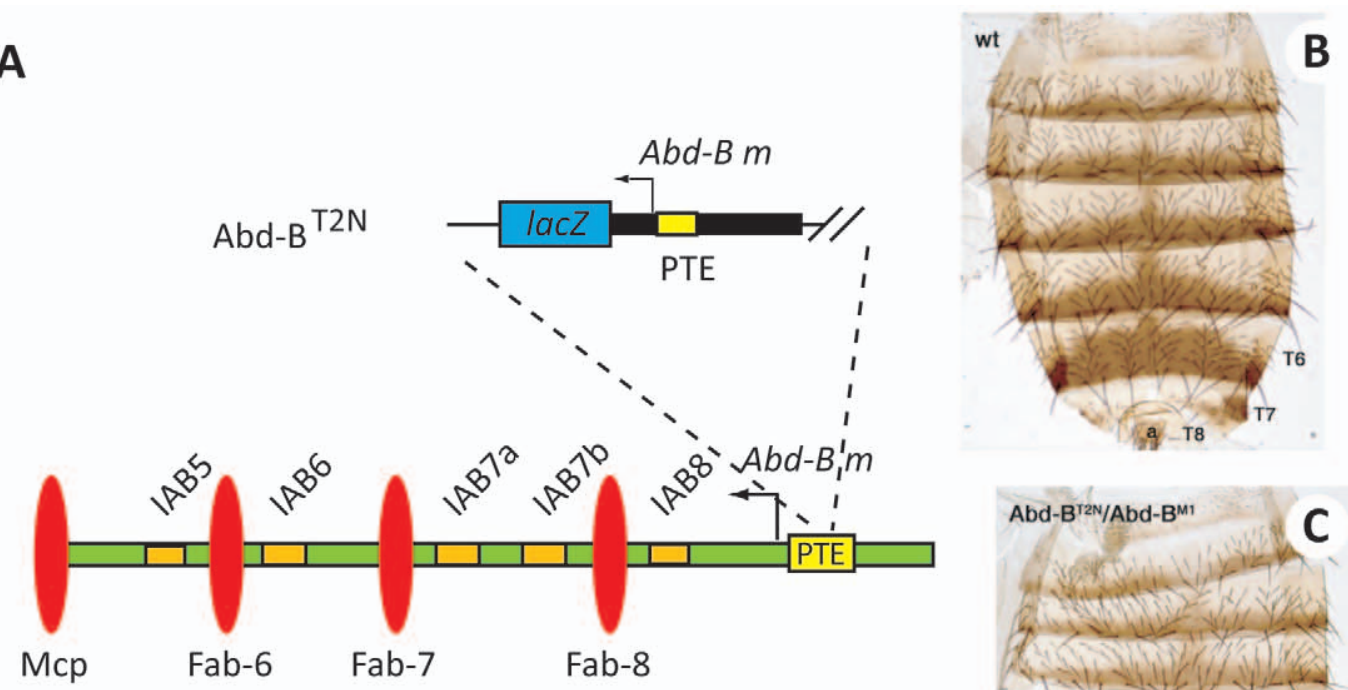

B

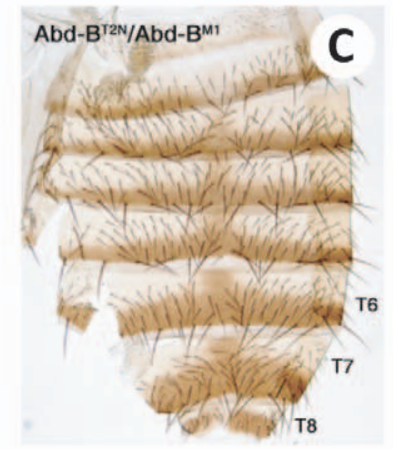

$i a b-5 \quad i a b-6$

$i a b-7$

$i a b-8$

(PS10)

(PS11)

(PS12)

(PS13)
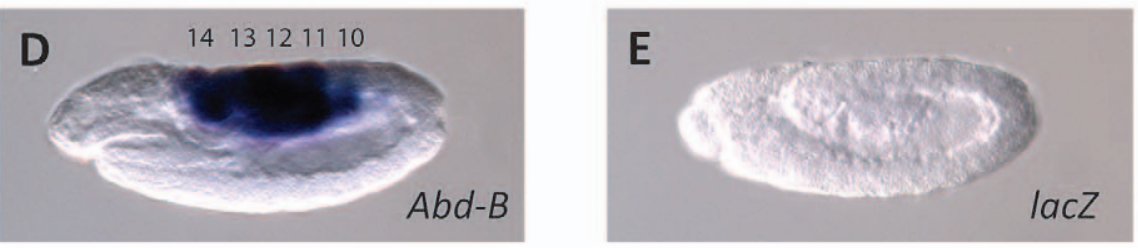

\section{WT $\left(\mathrm{yw}^{67}\right)$}
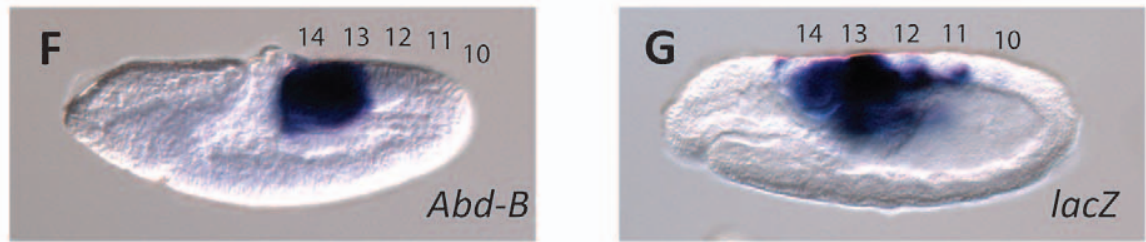

Abd-B

$\mathrm{T} 2 \mathrm{~N}$
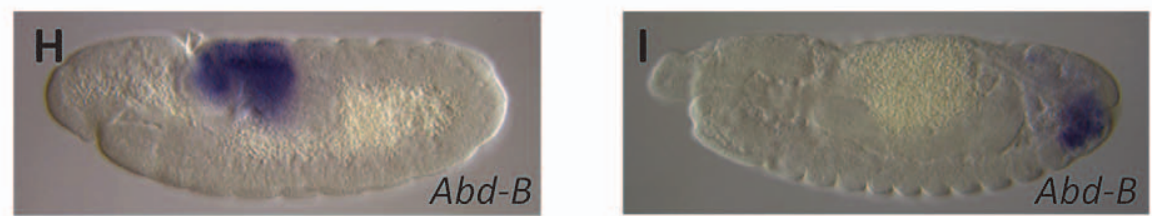

Figure 2. $P$ element insertion into the endogenous PTE results in homeotic transformation and loss of $A b d-B$ expression. (A) Schematic diagram of the $A b d-B^{T 2 N} P$ element line, showing the insertion site $-241 \mathrm{bp} 5^{\prime}$ of the $A b d-B m$ transcription start site in the endogenous PTE sequence. The same symbols and color scheme as in Fig. 1 indicate the neighboring cis-regulatory modules. (B) Dorsal cuticles were prepared from adult females. A normal abdominal pigmentation pattern is observed in wild-type (WT) adults. (C) In $A b d-B^{T 2 N} / A b d-B^{M 1}$ hemizygotes the seventh abdominal tergite (T7) is fully developed, indicating a transformation toward a more anterior abdominal segment identity. The eighth abdominal tergite (T8) also shows a partial transformation toward a more anterior identity. $A b d-B^{M 1}$ is a Class III null allele for both the $A b d-B m$ and $r$ transcripts $[15,27]$. $A b d-B$ gene expression pattern in wild-type (WT) (D) and $A b d-B^{T 2 N}$ (F) germ-band elongation stage 9 embryos. The staining patterns for the $A b d-B^{T 2 N}$ line show reduced $A b d-B$ expression in parasegments (PS) 10,11 and 12 at stage $9(\mathbf{F})$, stage 11 (H) and stage 13 (I) of development. The lacZ reporter gene is expressed in developing posterior regions of the $A b d-B^{T 2 N}$ embryos (G). Expression is strongest in PS13, although the pattern extends from PS 10-14 and is very similar to the endogenous $A b d-B$ transcription pattern. No lacZ expression is detectable in WT embryos (E). doi:10.1371/journal.pone.0016283.g002 
(Fig. 3). This was accomplished by performing an imprecise excision of the $P$ element insertion from the $A b d-B^{I D \mathcal{N}}$ mutant (Fig. $\mathrm{S} 1)$. The $A b d-B^{L D N}$ mutant was generated by a $P$ element replacement strategy and carries an insertion containing the GAL4 and white genes in the identical location within the PTE ( -241 bp relative to the $A b d-B$ transcription start site) as the $A b d-$ $B^{T 2 \mathcal{N}}$ line, but does not contain an ectopic copy of the PTE (Fig. 3a) [31]. In the $A b d-B^{L D N}$ line, both the white and GAL4 genes are only expressed clearly in PS13 and very weakly in PS14 in embryos (data not shown), indicating that the distantly located IAB5-7 enhancers in the BX-C are not activating transcription of either reporter gene. The $A b d-B^{L D \mathcal{N}}$ line was utilized to generate an imprecise excision mutant as it carries the readily detectable white reporter gene (Fig. 3a). As a result, adult flies in which $P$ element excision had occurred were easily identified by loss of red eye color (Fig. S1). PCR-based screening with primers from the proximal $A b d-B m$ promoter region and from $0.5 \mathrm{~kb}, 1 \mathrm{~kb}$ and $1.5 \mathrm{~kb} 5^{\prime}$ of the $A b d-B$ transcription start site was used to identify a $1.2 \mathrm{~kb}$ deletion in the $A b d-B^{\Delta \text { PTE-UP }}$ allele (Fig. $3 \mathrm{a}-\mathrm{c}$ ). The molecular nature of the deletion was characterized by sequencing and found to have removed $53 \mathrm{bp}$ of the PTE sequence $5^{\prime}$ of the original $P$ element insertion site, as well as $1134 \mathrm{bp}$ of endogenous genomic sequence $5^{\prime}$ of the defined PTE. In this $A b d-B^{\Delta \text { PTE-UP }}$ allele, a portion of the GAL4 gene from the original $P$ element remains, along with $202 \mathrm{bp}$ of the 3' PTE sequence (Fig. 3b).

Based on the $A b d-B$ expression observed in the $A b d-B^{T 2 N}$ insertion line, we hypothesized that the expression pattern of the endogenous $A b d-B$ gene from the $A b d-B^{\Delta \text { PTE-UP }}$ allele may also be perturbed. In situ hybridization with an RNA probe that can detect both the $m$ and $r$ transcripts shows that $A b d-B$ gene expression is lost specifically in PS10-12, but not in PS13-14 in germ-band elongation stage embryos generated from crosses of heterozygous balanced $A b d-$ $B^{\Delta \text { PTE-UP }}$ adults (Fig. 3d). Statistical analysis demonstrates that the number of embryos demonstrating this restricted $A b d-B$ expression pattern from both the $A b d-B^{\triangle \mathrm{PTE}-\mathrm{UP}}$ and $A b d-B^{L D \mathcal{N}}$ alleles is highly significant $(\mathrm{p}<0.01)$ when compared to the number of embryos with the WT $A b d-B$ expression pattern. This restricted pattern of $A b d-B$ expression persists at least through stage 13 of development (data not shown). One possible explanation for the loss of $A b d-B$ expression in PS10-12 is that the genomic region deleted in the $A b d-B^{\Delta \text { PTE-UP }}$ allele harbors a CRM capable of driving transcription in these specific parasegments. However, when tested in a transgenic reporter gene assay the $1.2 \mathrm{~kb}$ region does not exhibit embryonic enhancer activity (data not shown).

To confirm that mutations in the PTE only affect expression from the $A b d-B m$ promoter, but not the $A b d-B r$ promoter, in situ hybridization with a RNA probe (BPP, [32]) that specifically detects only the $A b d-B r$ transcript was performed on embryos collected from WT, $A b d-B^{L D N}$ and $A b d-B^{\Delta \text { PTE-UP }}$ balanced lines. The expression pattern of the $A b d-B r$ transcript was confirmed to be identical in the WT, $A b d-B^{L D \mathcal{N}}$ and $A b d-B^{\Delta \text { PTE-UP }}$ embryos, appearing only in PS14 of germ-band elongation stage embryos (Fig. 3d). These observations are consistent with the known pattern of expression from the $A b d-B r$ promoter [29] and confirm that the disruption to the PTE in these mutants only affects enhancermediated transcription from the $A b d-B m$ promoter (see discussion for more detail).

\section{Discussion}

\section{Parasegment-specific interactions between the IAB enhancers and $A b d-B$ promoter in the BX-C}

The absence of $A b d-B$ expression in PS10-12 of $A b d-B^{4 P T E-U P}$ germ-band elongation stage embryos is consistent with a loss of
IAB-enhancer directed expression from the $A b d-B m$ promoter [29]. This suggests that deletion of the $A b d-B$ promoter tethering sequence (PTE) and the neighboring $1.1 \mathrm{~kb} 5$ ' sequence in the $A b d-B^{A P T E-U P}$ line leads to a disruption of the long-range interactions between the $A b d-B m$ promoter and enhancers from the $i a b-5$, $i a b-6$ and $i a b-7$ regions in PS10, 11 and 12, respectively. For example, in PS12 of WT embryos the tethering sequences upstream of the $A b d-B m$ promoter enable the IAB7a and IAB7b embryonic enhancers to bypass the Fab-8 chromatin insulator and drive expression from the $A b d-B \mathrm{~m}$ promoter (Fig. 4a). In $A b d-$ $B^{\triangle P T E-U P}$ mutant embryos, removal of the tethering sequences appears to disrupt the ability of the IAB $7 \mathrm{a}$ and IAB7b enhancers to activate the $A b d-B m$ promoter, resulting in an absence of $A b d-B$ expression in PS12 (Fig. 4a and 3d). Similarly, the IAB6 and IAB5 enhancers are unable to bypass intervening insulators to activate $A b d-B m$ expression in PS11 and PS10 in $A b d-B^{A P T E-U P}$ embryos. In contrast to PS10-12, the specific enhancer-promoter interactions at the BX-C in PS13 appear to be intact in $A b d-B^{\triangle P T E-U P}$ embryos. In WT embryos the IAB8 embryonic enhancer, located $3^{\prime}$ of the $A b d-B$ gene, is solely responsible for directing expression from the $A b d-B m$ promoter in PS13 [33]. In $A b d-B^{A P T E-U P}$ mutant embryos the $A b d-B m$ transcript remains strongly expressed in PS13 (Fig. 3d). This result indicates that the interaction between the IAB8 enhancer and the $A b d-B m$ promoter may not require tethering activity, likely due to the physical proximity of the enhancer to the promoter and lack of an intervening chromatin insulator (Fig. 4a). However, given the partial transformation phenotype observed for PS13 in flies carrying a disruption of the PTE sequences (as in the case of the $A b d-B^{T 2 \mathcal{N}}$ allele, Fig. 2c) it remains possible that loss of PTE activity may be responsible for subtle effects on transcription of $A b d-B$ in PS13. In PS14, $A b d-B$ expression in germ-band elongation stage embryos is not driven by the $3^{\prime} \mathrm{IAB}$ enhancers, as it is initiated from the $r$ transcriptional start site located $5^{\prime}$ of the PTE sequence (Fig. 1) [29,34]. Consequently, expression of the $r$ transcript in PS14 is not perturbed by the loss of tethering activity in $A b d-B^{A P T E-U P}$ mutant embryos (Fig. 4a and 3d).

One possible outcome of the disruption of IAB enhancer interactions with the $A b d-B m$ promoter at the $A b d-B^{\triangle P T E-U P}$ allele may be the re-direction of those enhancers to the neighboring $a b d$ $A$ promoter in the BX-C. However, no change in the expression pattern of $a b d-A$ (extending from PS7 to PS13 in germ-band elongation stage embryos [35] could be detected from the $A b d^{-}$ $B^{4 P T E-U P}$ or $A b d-B^{\mathrm{LDN}}$ alleles (data not shown).

\section{Functional dissection of critical sequences at the PTE}

The functional activity of the PTE at the endogenous BX-C prompts the question of exactly what sequences associated with the PTE are necessary to confer promoter-enhancer tethering. Our earlier studies demonstrated that a 255 bp region located between -40 and $-294 \mathrm{bp} 5^{\prime}$ of the $A b d-B m$ transcription start site is sufficient to tether the IAB 5 enhancer to an ectopic promoter in a transgene competition assay (Fig. 4b) [16]. In contrast, an approximately $200 \mathrm{bp}$ region extending from -100 to +100 relative to the $A b d-B m$ transcriptional start site is not sufficient to mediate promoter-enhancer tethering when tested in similar transgenic assays [36]. In agreement with this observation, the $202 \mathrm{bp}$ of sequence from the $3^{\prime}$ end of the PTE remaining at the endogenous BX-C in the $A b d-B^{\triangle P T E-U P}$ allele is also not sufficient to mediate tethering of the IAB enhancers to the $A b d-B m$ promoter (Fig. 4b). A previous study by Sipos and colleagues, utilizing an $A b d-B$ mutant allele harboring a small deletion at the $A b d-B m$ promoter region and a deletion at the $i a b-7$ region carried on opposite chromosomes resulted in $A b d-B$ transcriptional activity 
A

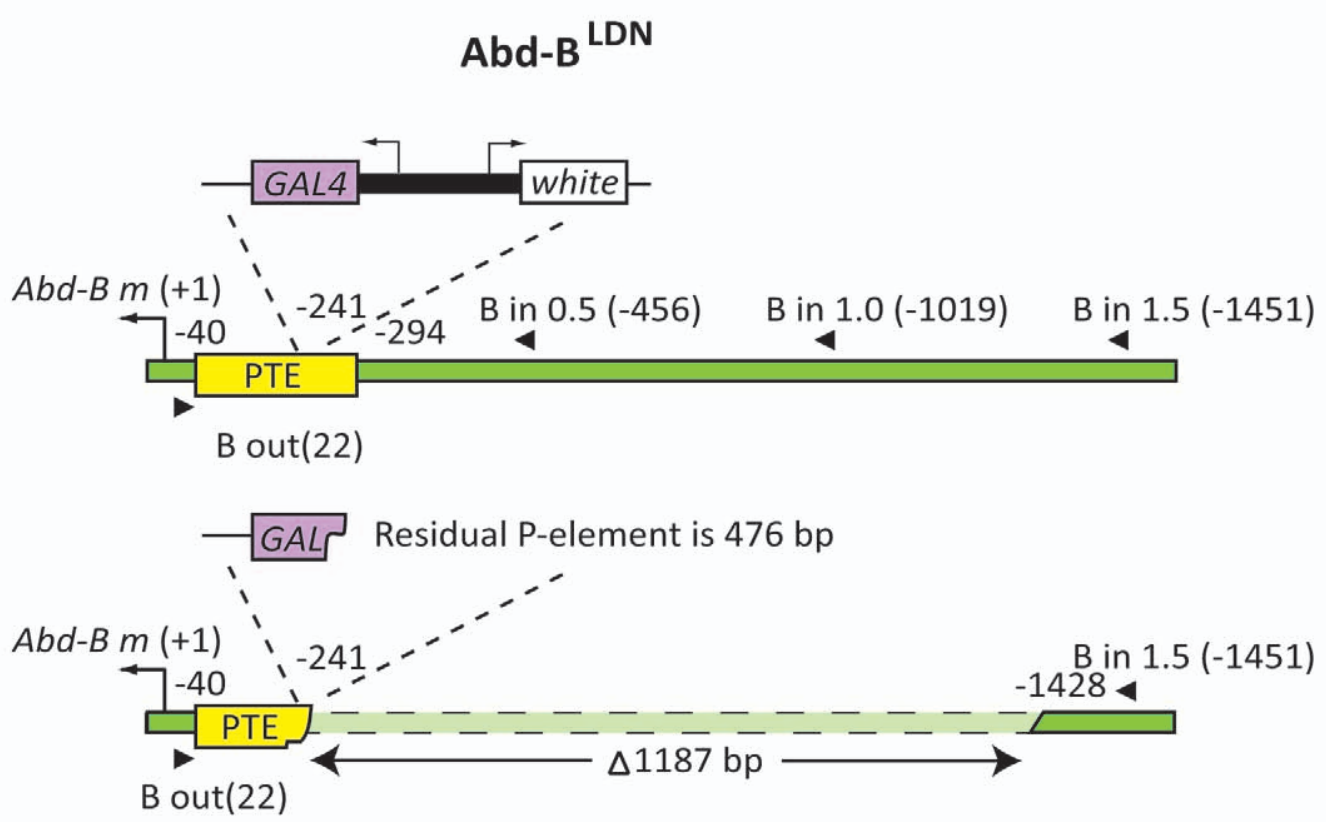

B Abd-B $\triangle$ PTE-UP

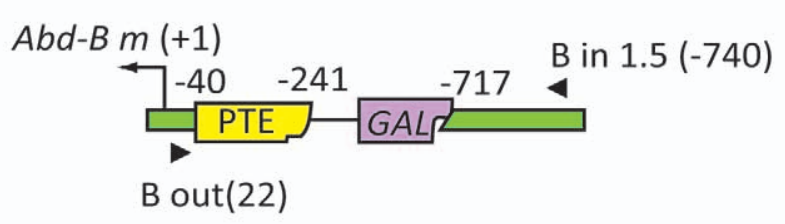

C

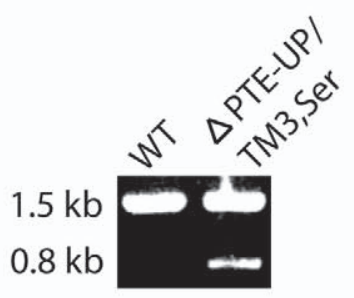

D

$$
\text { WT (yw } \left.{ }^{67}\right)
$$

Abd-B

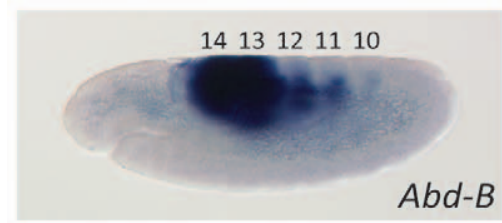

$14 \quad 13 \quad 121110$
1413121110

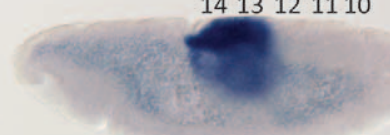

$A b d-B$
Abd-B $\triangle \mathrm{PTE}-\mathrm{UP}$

$A b d-B r$

$A b d-B r$

$A b d-B r$

Figure 3. Deletion of the PTE and upstream sequences at the endogenous BX-C disrupts $A b d-B$ expression. (A) The $A b d-B^{L D N}$ mutant features a $P$ element inserted within the PTE (yellow box) $(-241$ bp relative to the $A b d-B$ transcription start site (black arrow)). The $P$ element contains GAL4 (purple box) and white (white box) reporter genes. PCR primers (black triangles) designed against the $A b d-B$ promoter region and $0.5 \mathrm{~kb}, 1 \mathrm{~kb}$ and $1.5 \mathrm{~kb}$ upstream of the $A b d-B \mathrm{~m}$ transcription start site were used to characterize the approximately $1.2 \mathrm{~kb}$ deletion (dashed box) in the $A b d-$ $B^{\triangle P T E-U P}$ mutant generated by imprecise excision of the $P$ element. (B) The resulting $A b d-B^{\triangle P T E-U P}$ deletion removes 53 bp of the PTE sequence $5^{\prime}$ of the original insertion site and an additional 1134 bp of endogenous sequence $5^{\prime}$ of the PTE. A 476 bp portion of the GAL4 coding sequence (purple polygon) from the original $P$ element remains, as well as 202 bp of the $3^{\prime}$ PTE sequence (yellow polygon). (C) Primers designed against the $A b d-B$ promoter region and $1.5 \mathrm{~kb}$ upstream of the $A b d-B$ transcription start site were used to genotype adult flies carrying the deletion allele by amplifying the $1.5 \mathrm{~kb}$ wild-type (WT) band and the $0.8 \mathrm{~kb} A b d-B^{\Delta \mathrm{PTE}-\mathrm{UP}}$ band in balanced $A b d-B^{\Delta \mathrm{PTE}-\mathrm{UP}}$ ( $\left.\triangle \mathrm{PTE}-\mathrm{UP} / T M 3, S e r\right)$ individuals. (D) In situ hybridization 
with an RNA probe that can detect both the $A b d-B m$ and $r$ transcripts ( $A b d-B$ ) shows WT expression in parasegments (PS) $10-14$ in yw ${ }^{67}$ embryos, while expression is found only in PS13-14 in a portion of embryos collected from crosses of balanced $A b d-B^{\triangle \mathrm{PTE}-U \mathrm{UP}}$ and $A b d-B^{\mathrm{LDN}}$ lines. In situ hybridization with a probe specifically designed against the $A b d-B r$ transcript ( $A b d-B r$ ) detects identical patterns of expression in PS14 of germ-band elongation stage embryos collected from WT, mutant $A b d-B^{L D N}$ and $A b d-B^{\triangle P T E-U P}$ balanced lines. doi:10.1371/journal.pone.0016283.g003

[25]. This indicates that a trans interaction can occur between the $i a b-7$ regulatory region and the remaining $A b d-B$ promoter region across chromosomes. The same genetic complementation test using the $i a b-7$ mutant allele and the $A b d-B^{D 18}$ deletion allele (which removes approximately $8 \mathrm{~kb}$ of sequence $5^{\prime}$ of the $A b d-B m$ transcription start site) on opposite chromosomes showes an increase in trans-mediated $A b d-B$ activity [25]. These results indicate that additional sequences located $5^{\prime}$ of the 255 bp PTE may also contribute to tethering activity. A functional role for the sequences in the $5^{\prime}$ end of the PTE and neighboring upstream genomic regions is supported by the loss of enhancer-directed $A b d-$ $B m$ transcript expression at the $A b d-B^{A P T E-U P}$ allele after deletion of the 5' $53 \mathrm{bp}$ region in the PTE and $1134 \mathrm{bp}$ of sequence located $5^{\prime}$ of the defined PTE (Fig. 4b). The loss of $A b d-B$ expression observed in the $A b d-B^{A P T E-U P}$ mutant is consistent with the previously reported molecular phenotype of $A b d-B m$ mutants [27] and confirms the role of the PTE and associated 5' sequences in mediating specific IAB enhancer-directed expression of the $A b d$ $B m$ transcript.

\section{Promoter tethering as a general regulatory mechanism}

Additional examples of genetic complexes in which long-range interactions between CRMs have been characterized include the human beta-globin locus [37], the Drosophila Antennapedia complex [38,39] and mouse HoxD complex [3]. In the large genomes of vertebrates, where extensive global control regions have been identified, promoter-enhancer tethering is emerging as a critical general mechanism for regulation of gene expression [3,37]. More recently, in Drosophila, other PTE sequences have been located in the even-skipped [40] and engrailed [41] loci that mediate enhancer-promoter communication. The long-range CRM communication in these critical developmental genes has been shown to be dynamic, changing through the course of development in some cases [40] and perhaps even capable of mediating the evolution of novel patterns of gene expression in different insect species [42].

Our current model for the molecular function of the PTE in the Drosophila BX-C is that regulatory interactions that enable the PTE to tether the IAB enhancers to the $A b d-B m$ promoter in specific parasegments during embryonic development may be mediated by chromatin looping [17]. A number of studies have indicated that chromatin looping may facilitate promoterenhancer tethering through the action of different transcription factors. For example, the sea urchin GCF1 protein is able to form higher order multimeric loop structures when added to target site oligonucleotides in vitro [43]. The abundant mammalian transcription factor $\mathrm{Spl}$ has also been shown to form multimers and to strongly facilitate in vivo activation of a promoter by distantly located enhancer CRMs [44,45]. More recently, molecular studies have used high-magnification confocal imaging and 2D RNA fluorescence in situ hybridization (FISH) to visualize specific physical associations between distantly located cisregulatory sequences in the nucleus at the human beta-globin locus [37].

Direct evidence for chromatin looping at the Drosophila BX-C comes from a study examining physical chromosomal interactions using probes designed against the IAB 5 and IAB 8 enhancers and a promoter-proximal region upstream of the $A b d-B m$ transcription unit, containing the PTE sequence [46]. In a portion of nuclei taken from the eighth abdominal segment region of a germ-band elongation stage embryo, FISH signals from the $A b d-B m$ promoter region co-localize with signals from the distal IAB5 enhancer, while the more proximal IAB8 enhancer remains distantly located and disassociated [46]. This result suggests that physical associations may indeed occur between the IAB5 enhancer and the $5^{\prime}$ upstream region of the $A b d-B m$ promoter to facilitate expression of the $A b d-B m$ transcript. These data support our model that tethering mediates the interaction of long-range enhancers (such as IAB5) to the $A b d-B m$ promoter, but that it is not required for the functional interaction of the $3^{\prime}$ proximal IAB8 enhancer to the $A b d-B m$ promoter.

In our current model the PTE may bind sequence-specific protein factors which interact with complementary factors bound near the IAB enhancers, allowing a molecular bridge or loop to form between the $A b d-B m$ promoter and the IAB enhancers [17]. This model does not exclude the possibility that additional molecular interactions between known regulatory regions in the BX-C, including insulator and PTS sequences, may also mediate enhancer-promoter communication. In future studies other techniques, such as three-dimensional chromatin conformation capture (3C) [47] and Dam methylase identification [48], will be well suited to more fully elucidate the nature of such interactions and the molecular mechanisms of PTE function. In addition, molecular dissection of the functional sequences within the PTE and $5^{\prime}$ associated sequences in transgenic tethering assays and the biochemical identification of putative sequence-specific DNAbinding trans factors that may mediate the PTE activity will be essential.

\section{Materials and Methods}

\section{Genetic insertion lines}

The $A b d-B^{T 2 \mathcal{N}}$ and $A b d-B^{L D \mathcal{N}} P$ element fly lines inserted -241 bp $5^{\prime}$ of the $A b d-B$ m transcription start site were provided by Ernesto Sanchez-Herrero [27,31].

\section{$P$ element imprecise excision from the $A b d-B^{L D N}$ line}

$A b d-B^{L D N}$ flies were crossed with a transposase expressing line $(\Delta 2-3)$ carrying a Stubble $(\mathrm{Sb})$ dominant phenotypic marker (BL Stock 1798) in Cross 1 (Fig. S1). Cross 1 male progeny were screened for $\mathrm{Sb}$ (marking the presence of $\Delta 2-3$ transposase) and variegated red eye color (rather than white eye color). The selected Cross 1 males were crossed with female with D, a dominant marker, and a TM3 balancer chromosome carrying Serrate (Ser), a dominant phenotypic marker (BL Stock 7198) in Cross 2 (Fig. $\mathrm{S} 1$ ). The male Cross 2 progeny were screened for Ser (marking the presence of the TM3 balancer), white eyes (indicating excision of the $A b d-B-G A L 4^{L D N} P$ element construct), and the absence of $\mathrm{Sb}$ (indicating loss of the $\Delta 2-3$ transposase) as well as against $\mathrm{D}$. The selected Cross 2 male flies were crossed with BL Stock 7198 flies again in Cross 3 (Fig. S1). After a few days, the male parents were recovered from Cross 3 vials. PCR amplification of the Abd-B region with primers located $0.5 \mathrm{~kb}, 1 \mathrm{~kb}$, and $1.5 \mathrm{~kb}$ upstream of the $A b d-B$ transcription start site on the genomic DNA prepared from approximately 100 selected Cross 2 male flies was used to detect deletions in the PTE sequence. 
A
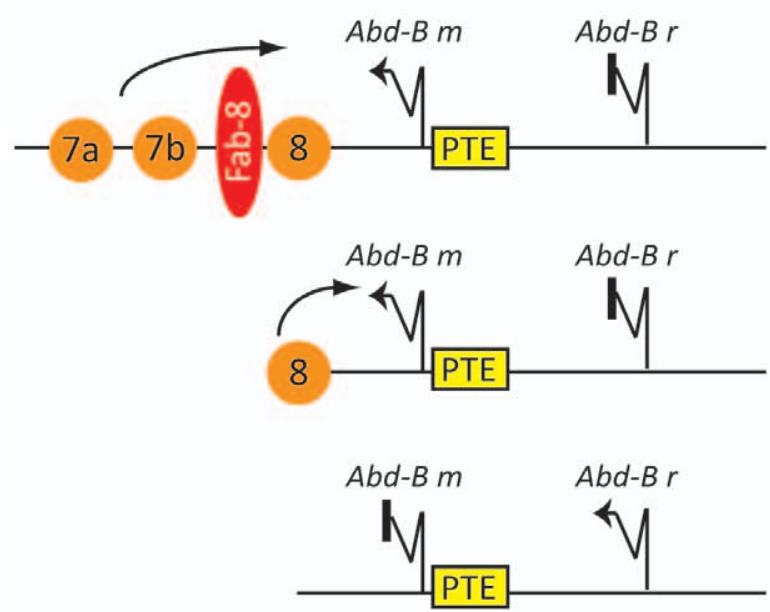

PS12
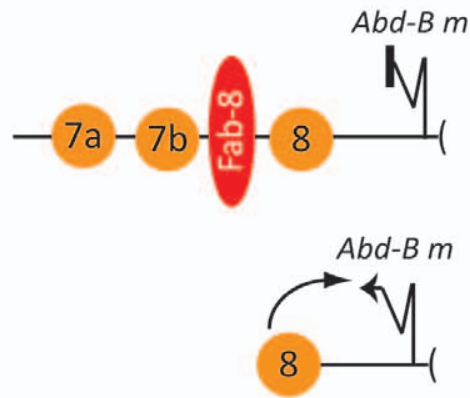

PS14
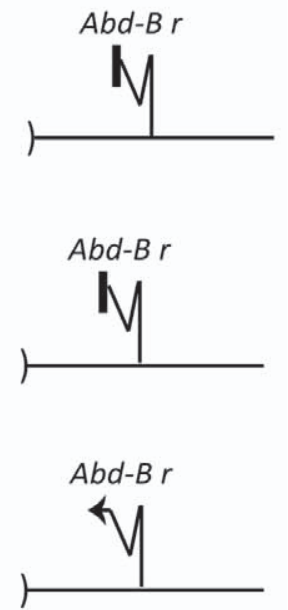

$\triangle \mathrm{PTE}-U \mathrm{P}$

PS13

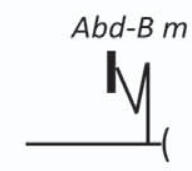

B

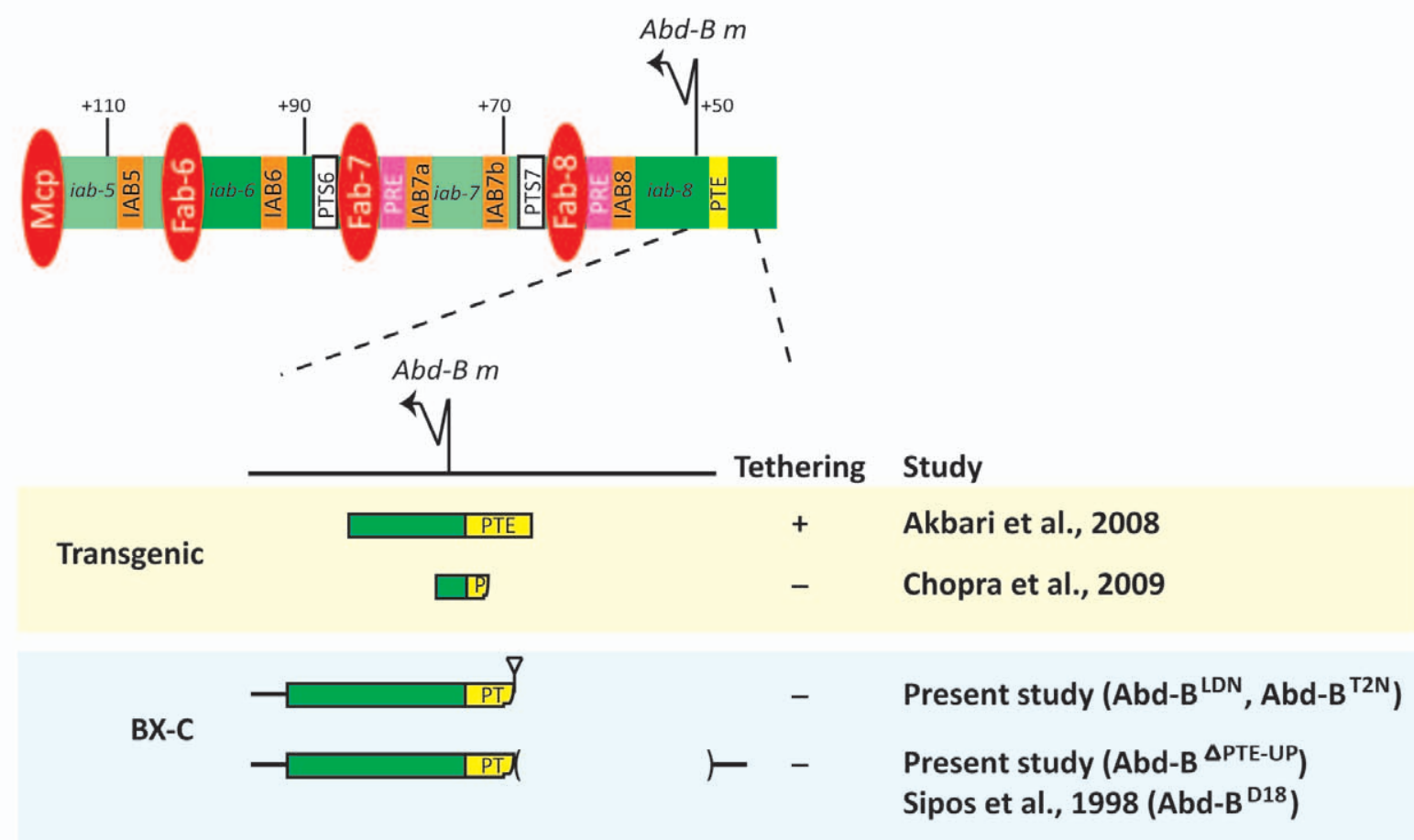

Figure 4. PTE sequence is required for specific promoter-enhancer tethering at the $A b d-B$ gene. (A) $A b d-B$ transcription is disrupted in PTE mutants. In wild-type (WT) germ-band elongation stage embryos expression from the $A b d-B m$ promoter is observed in parasegments (PS) 10-13 and from the $A b d-B r$ promoter in PS14 [29]. The loss of $A b d-B$ expression in PS10-12 in $A b d-B^{A P T E-U P}$ ( $\triangle P T E-U P$ ) embryos is consistent with a disruption of expression from the $A b d-B m$ promoter. In PS12 of WT embryos, the tethering sequences upstream of the $A b d-B m$ promoter enable the IAB7 $a$ and IAB7b enhancers ( $7 \mathrm{a}$ and $7 \mathrm{~b}$, orange circles) to act over the long-range of intervening sequence and bypass the Fab-8 chromatin insulator (red ellipse) and drive expression from the $A b d-B m$ promoter. In PS12 of $A b d-B{ }^{A P T E-U P}$ mutants the removal of the tethering sequences disrupts the ability of the IAB7 enhancers to activate the $A b d-B m$ promoter across the intervening Fab-8 insulator. A similar disruption of promoter-enhancer tethering activity at $A b d-B^{4 P T E-U P}$ is also seen in PS11 (IAB6 enhancer) and PS10 (IAB5 enhancer). In PS13 of both WT and Abd- $B^{4 P T E-U P}$ embryos, IAB8 $(8$, orange circle) is able, due to its physical proximity and lack of intervening chromatin insulator, to drive expression from the $A b d-B m$ promoter. In PS14 of both WT and $A b d-B^{A P T E-U P}$ embryos, $A b d-B r$ is transcribed independently of IAB embryonic enhancer activity and represses transcription from the $A b d-B m$ promoter [28]. (B) Functional dissection of critical $\boldsymbol{A} b \boldsymbol{d}-\boldsymbol{B}$ tethering sequences. Schematic diagram of the regulatory region of the $A b d o m i n a l-B$ gene shows enhancers (orange boxes) in the infraabdominal regions iab-5 to iab-8 (green) that are responsible for activating expression of the $A b d-B$ gene in abdominal PS 10-13 during embryogenesis. Color scheme for other types of cis-regulatory modules is same as in Figure 1. Transgenic studies (highlighted in yellow box) show that a $1.4 \mathrm{~kb}$ of sequence from +1228 to -294 , (including the 255 bp PTE sequence located $40 \mathrm{bp} 5^{\prime}$ of the $A b d-B \mathrm{~m}$ transcription start site), is sufficient for promoter-enhancer tethering [16]. Additional transgenic studies have shown that an approximately $200 \mathrm{bp}$ sequence spanning the $A b d-B m$ transcription start site $(+100$ to -100$)$ is not sufficient for tethering activity [36]. $P$ element insertion at the endogenous BX-C (as in the $A b d-B^{L D N}$ or $A b d-B^{T 2 N}$ mutations) demonstrates that the 242 bp sequence $5^{\prime}$ of the $A b d-B m$ transcription 
site (from +1 to -241 ), including 202 bp of the identified PTE sequence, is not sufficient to tether the distally located IAB5, 6 and 7 enhancers in vivo. Deletion of an approximately $1.2 \mathrm{~kb}$ sequence $(-241$ to -1428$)$ which include $53 \mathrm{bp}$ of the $5^{\prime}$ region of the PTE and neighboring upstream sequences in the $A b d-B^{\Delta P T E-U P}$ mutant also results in a loss of tethering. The 53 bp region of the PTE may therefore be important for functional tethering. A potential tethering role at the endogenous BX-C for the sequences $5^{\prime}$ of the PTE is also suggested by earlier genetic complementation studies in the $A b d-B^{D 18}$ allele [25].

doi:10.1371/journal.pone.0016283.g004

Primers used:

$A b d-B$ promoter out: $5^{\prime}$-CGA CAA CAT ATG CAG ATG GCT-3'

$A b d-B$ promoter $0.5 \mathrm{~kb}$ in: 5 '-AAG TGG GAT ACG ATG TTT-3'

$A b d-B$ promoter $1 \mathrm{~kb}$ in: $5^{\prime}$-TGC CTT TGG AAG TGA GAC $\mathrm{AA}-3^{\prime}$

$A b d-B$ promoter $1.5 \mathrm{~kb}$ in: $5^{\prime}$-GGA AAT AGA TTG CGG CAG TTA A-3'

The PCR products were ligated into pGEM-T Easy vector (Promega) and sequenced using T3 and SP6 sequencing primers. Progeny from Cross 3 vials seeded with a male parent exhibiting a disruption of the PTE were screened against D and for Ser and self-crossed to generate a balanced mutant line.

\section{In situ analysis of $a b d-A$ and $A b d-B$ expression}

In situ hybridization probes to detect transcription of $A b d-B$ and $a b d-A$ were PCR-amplified using $D$. melanogaster yw ${ }^{67}$ adult genomic DNA as a template. The previously described DNA sequences for the Bexon region (exon 8 of the D. melanogaster $A b d-B$ gene), BPP (specific to the $r$ transcript) and Aexon region [32] were PCR amplified and cloned into pGEMT-Easy (Promega). PCR primer sequences were as follows:

Bexon s: 5'-GAACAAGAAGAAGTCACAGG-3';

Bexon as: 5'-TAGGCATAGGTGTAGGTGTAGG-3';

BPP s: 5'-TATTATTCGTCTCCAGTCGC-3';

BPP as: 5'-CTCAGATTGATGGTGGTGGTGG-3';

Aexon s: 5' - CACCAACAGCAGCAACAACAGC-3' (173566);

Aexon as: 5' - CATTGTATTCAAGGGTTGGC-3' (174756);

Antisense RNA probes (relative to the direction of $A b d-B$ and $a b d-A$ transcription) were prepared using a digoxigenin (DIG) RNA-labeling kit (Roche, Gipf-Oberfrick, Switzerland). Embryos from each of the wild-type $D$. melanogaster, and mutant $A b d-B^{T 2 N}$, $A b d-B^{L D N}$ and $A b d-B^{\triangle P T E-U P}$ lines were collected, fixed and hybridized with the appropriate probes as previously described [32]. Anti-sense Bexon RNA probes enable the detection of both the $m$ and $r$ transcript and anti-sense BPP RNA probes enable the specific detection of only the $A b d-B r$ transcript [32].

\section{Supporting Information}

Figure S1 Grosses to generate Abd-B ${ }^{\Delta P T E-U P}$ mutant. $(A)$ Schematic diagram of the $A b d-B^{L D \mathcal{N}} P$ element insertion line,

\section{References}

1. Caplan AI, Ordahl CP (1978) Irreversible gene repression model for control of development. Science 201: 120-130.

2. Lewis EB (1978) A gene complex controlling segmentation in Drosophila. Nature 276: 565-570.

3. Spitz F, Gonzalez F, Duboule D (2003) A Global Control Region Defines a Chromosomal Regulatory Landscape Containing the HoxD Cluster. Cell 113: 405-417.

4. Osborne CS, Chakalova L, Brown KE, Carter D, Horton A, et al. (2004) Active genes dynamically colocalize to shared sites of ongoing transcription. Nat Genet 36: $1065-1071$.

5. Dorsett D (1999) Distant liaisons: long-range enhancer-promoter interactions in Drosophila. Curr Opin Genet Dev 9: 505-514.

6. Kellum R, Elgin SC (1998) Chromatin boundaries: punctuating the genome. Curr Biol 8: R521-524.

7. Mihaly J, Hogga I, Barges S, Galloni M, Mishra RK, et al. (1998) Chromatin domain boundaries in the Bithorax complex. Cell Mol Life Sci 54: 60-70. showing the insertion site -241 bp $5^{\prime}$ of the $A b d-B m$ transcription start site in the endogenous PTE sequence. The $P$ element insertion in the $A b d-B^{L D \mathcal{N}}$ line contains GAL4 (purple box) and white (white box) reporter genes. The same symbols and color scheme as shown in Fig. 1 are used to show the cis-regulatory modules. $(\mathbf{B})$ $A b d-B^{L D N}$ flies were crossed with a transposase expressing line $(\Delta 2-$ 3) carrying Stubble (Sb) (BL Stock 1798) (Cross 1). Cross 1 male progeny were screened for $\mathrm{Sb}$ and variegated red eye color (rather than white eye color). (G) The selected Cross 1 males were crossed with a female with $\mathrm{D}$, a dominant marker, and a TM3 balancer chromosome carrying Serrate (Ser), a dominant phenotypic marker (BL Stock 7198) (Cross 2). Cross 2 male progeny were screened for Ser, white eyes (indicating excision of the GAL4 LDN $P$ element construct), and the absence of $\mathrm{Sb}$ and $\mathrm{D}$. (D) The selected Cross 2 male flies were crossed with BL Stock 7198 flies again (Cross 3). After a few days, the male parents were recovered from the Cross 3 vials. PCR amplification of the $A b d-B$ promoter region with primers (black triangles) located $0.5 \mathrm{~kb}, 1 \mathrm{~kb}$, and $1.5 \mathrm{~kb}$ upstream of the $A b d-B$ transcription start site on the genomic DNA prepared from these selected Cross 2 male flies was used to detect deletions in the PTE sequence. Progeny originating from Cross 3 vials seeded with a male parent exhibiting a disruption of the PTE ( $\triangle \mathrm{PTE})$ were recovered and screened against D and for Ser. (E) These selected Cross 3 progeny were then self-crossed to generate the $A b d-B^{\Delta \text { PTE-UP }} / T M 3$,Ser balanced line.

(TIF

\section{Acknowledgments}

The authors would like to thank Ernesto Sanchez-Herrero for providing the $A b d-B^{T 2 N}$ and Abd-B ${ }^{\mathrm{LDN}}$ lines. We would also like to thank Karl G. Johnson and Kathleen M. Beckingham for their helpful advice on the imprecise $P$ element excision.

\section{Author Contributions}

Conceived and designed the experiments: MCWH BJS RAD. Performed the experiments: MCWH BJS OSA EB RAD. Analyzed the data: MCWH BJS OAS EB RAD. Contributed reagents/materials/analysis tools: MCWH BJS RAD. Wrote the paper: MCWH BJS RAD.

8. Ohtsuki S, Levine M, Cai HN (1998) Different core promoters possess distinct regulatory activities in the Drosophila embryo. Genes \& Development 12: $547-556$.

9. Bell AC, Felsenfeld G (2000) Methylation of a CTCF-dependent boundary controls imprinted expression of the Igf2 gene. Nature 405: 482-485.

10. Celniker S, Drewell RA (2007) Chromatin looping mediates boundary element promoter interactions. BioEssays 29: 7-10.

11. Bushey AM, Dorman ER, Corces VG (2008) Chromatin insulators: regulatory mechanisms and epigenetic inheritance. Molecular Cell 10: 1-9.

12. Wallace JA, Felsenfeld G (2007) We gather together: insulators and genome organization. Curr Opin Genet Dev 17: 400-407.

13. Bender W, Akam M, Karch F, Beachy PA, Peifer M, et al. (1983) Molecular Genetics of the Bithorax Complex in Drosophila melanogaster. Science 221: 23-29.

14. Martin CH, Mayeda CA, Davis CA, Ericsson CL, Knafels JD, et al. (1995) Complete sequence of the bithorax complex of Drosophila. Proceedings of the National Academy of Sciences of the United States of America 92: 8398-8402. 
15. Karch F, Weiffenbach B, Peifer M, Bender W, Duncan I, et al. (1985) The abdominal region of the bithorax complex. Cell 43: 81-96.

16. Akbari OS, Bae E, Johnsen H, Villaluz A, Wong D, et al. (2008) A novel promoter-tethering element regulates enhancer-driven gene expression at the bithorax complex in the Drosophila embryo. Development 135: 123-131.

17. Akbari OS, Schiller BJ, Goetz SG, Ho MCW, Bae E, et al. (2007) The Abdominal-B promoter tethering element mediates promoter-enhancer specificity at the Drosophila bithorax complex. Fly 1: 337-339.

18. Mihaly J, Barges S, Sipos L, Maeda R, Cleard F, et al. (2006) Dissecting the regulatory landscape of the Abd-B gene of the bithorax complex. Development 133: 2983-2993.

19. Zhou J, Ashe H, Burks C, Levine M (1999) Characterization of the transvection mediating region of the abdominal-B locus in Drosophila. Development 126 : 3057-3065.

20. Karch F, Galloni M, Sipos L, Gausz J, Gyurkovics H, et al. (1994) Mcp and Fab7: molecular analysis of putative boundaries of cis-regulatory domains in the bithorax complex of Drosophila melanogaster. Nucleic Acids Research 22: 3138-3146.

21. Pérez-Lluch S, Cuartero S, Azorín F, Espinàs ML (2008) Characterization of new regulatory elements within the Drosophila bithorax complex. Nucleic Acids Res 36: 6926-6933.

22. Gyurkovics H, Gausz J, Kummer J, Karch F (1990) A new homeotic mutation in the Drosophila bithorax complex removes a boundary separating two domains of regulation. The Embo Journal 9: 2579-2585.

23. Lin Q Wu D, Zhou J (2003) The promoter targeting sequence facilitates and restricts a distant enhancer to a single promoter in the Drosophila embryo. Development 130: 519-526.

24. Lin O, Chen O, Lin L, Smith S, Zhou J (2007) Promoter targeting sequence mediates enhancer interference in the Drosophila embryo. Proc Natl Acad Sci U S A 104: 3237-3242.

25. Sipos L, Mihaly J, Karch F, Schedl P, Gausz J, et al. (1998) Transvection in the Drosophila Abd-B domain: extensive upstream sequences are involved in anchoring distant cis-regulatory regions to the promoter. Genetics 149: 1031-1050.

26. Sipos L, Gyurkovics H (2005) Long-distance interactions between enhancers and promoters. The case of the Abd-B domain of the Drosophila bithorax complex. FEBS Journal 272: 3253-3259.

27. Estrada B, Casares F, Busturia A, Sanchez-Herrero E (2002) Genetic and molecular characterization of a novel iab-8 regulatory domain in the Abdominal-B gene of Drosophila melanogaster. Development 129: 5195-5204.

28. Casanova J, Sanchez-Herrero E, Morata G (1986) Identification and characterization of a parasegment specific regulatory element of the abdominal-B gene of drosophila. Cell 47: 627-636.

29. Sánchez-Herrero E, Crosby MA (1988) The Abdominal-B gene of Drosophila melanogaster: overlapping transcripts exhibit two different spatial distributions. EMBO J 7: 2163-2173.

30. Delorenzi M, Bienz M (1990) Expression of Abdominal-B homeoproteins in Drosophila embryos. Development 108: 323-329.

31. de Navas L, Foronda D, Suzanne M, Sanchez_Herrero E (2006) A simple and efficient method to identify replacements of P-lacZ by P-Gal4 lines allows obtaining Gal4 insertions in the bithorax complex of Drosophila. Mechanisms of Development 123: 860-867.
32. Bae E, Calhoun VC, Levine M, Lewis EB, Drewell RA (2002) Characterization of the intergenic RNA profile at abdominal-A and Abdominal-B in the Drosophila bithorax complex. PNAS 99: 16847-16852.

33. Ho MC, Johnsen H, Goetz SE, Schiller BJ, Bae E, et al. (2009) Functional evolution of cis-regulatory modules at a homeotic gene in Drosophila. PLoS Genetics 5: e1000709.

34. Boulet AM, Lloyd A, Sakonju S (1991) Molecular definition of the morphogenetic and regulatory functions and the cis-regulatory elements of the Drosophila Abd-B homeotic gene. Development 111: 393-405.

35. Macias A, Casanova J, Morata G (1990) Expression and regulation of the abd-A gene of Drosophila. Development 110: 1197-1207.

36. Chopra VS, Cande J, Hong JW, Levine M (2009) Stalled Hox promoters as chromosomal boundaries. Genes and Development 23: 1505-1509.

37. Patrinos GP, de Krom M, de Boer E, Langeveld A, Imam AMA, et al. (2004) Multiple interactions between regulatory regions are required to stabilize an active chromatin hub. Genes Dev 18: 1495-1509.

38. Calhoun VC, Stathopoulos A, Levine M (2002) Promoter-proximal tethering elements regulate enhancer-promoter specificity in the Drosophila Antennapedia complex. Proceedings of the National Academy of Sciences of the United States of America 99: 9243-9247.

39. Calhoun VC, Levine M (2003) Long-range enhancer-promoter interactions in the Scr-Antp interval of the Drosophila Antennapedia complex. Proceedings of the National Academy of Sciences of the United States of America 100: 9878-9883.

40. Fujioka M, Wu X, Jaynes JB (2009) A chromatin insulator mediates transgene homing and very long-range enhancer-promoter communication. Development 136: 3077-3087.

41. Kwon D, Mucci D, Langlais K, Americo JL, DeVido SK, et al. (2009) Enhancer-promoter communication at the Drosophila engrailed locus. Development 136: 3067-3075.

42. Cande J, Chopra VS, Levine M (2009) Evolving enhancer-promoter interactions within the tinman complex of the flour beetle, Tribolium castaneum. Development 136: 3153-3160.

43. Zeller RW, Griffith JD, Moore JG, Kirchhamer CV, Britten RJ, et al. (1995) A multimerizing transcription factor of sea urchin embryos capable of looping DNA. PNAS 92: 2989-2993.

44. Su W, Jackson S, Tjian R, Echols H (1991) DNA looping between sites for transcriptional activation: self-association of DNA-bound Sp1. Genes \& Development 5: 820-826.

45. Mastrangelo IA, Courey AJ, Wall JS, Jackson SP, Hough PV (1991) DNA looping and Sp1 multimer links: a mechanism for transcriptional synergism and enhancement. Proceedings of the National Academy of Sciences of the United States of America 88: 5670-5674

46. Ronshaugen M, Levine M (2004) Visualization of trans-homolog enhancerpromoter interactions at the Abd-B Hox locus in the Drosophila embryo. Dev Cell 7: 925-932.

47. Hagège H, Klous P, Braem C, Splinter E, Dekker J, et al. (2007) Quantitative analysis of chromosome conformation capture assays (3C-qPCR). Nat Protoc 2: $1722-1733$.

48. Cleard F, Moshkin Y, Karch F, Maeda RK (2006) Probing long-distance regulatory interactions in the Drosophila melanogaster bithorax complex using Dam identification. Nature Genetics 38: 931-935. 